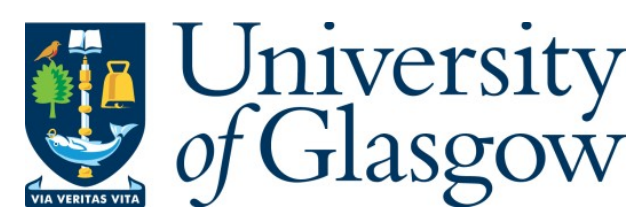

Mustafa, H. A., Shakir, M. Z., Imran, M. A., and Tafazolli, R. (2015) Distance Based Cooperation Region for D2D Pair. In: 2015 IEEE 81st Vehicular Technology Conference (VTC Spring), Glasgow, Scotland, 11-14 May 2015, ISBN 9781479980888.

There may be differences between this version and the published version. You are advised to consult the publisher's version if you wish to cite from it.

http://eprints.gla.ac.uk/133192/

Deposited on: 22 December 2016

Enlighten - Research publications by members of the University of Glasgow http://eprints.gla.ac.uk 


\title{
Distance Based Cooperation Region for D2D Pair
}

\author{
Hafiz A. Mustafa, Muhammad Z. Shakir, Muhammad A. Imran, and Rahim Tafazolli
}

\begin{abstract}
Device-to-device (D2D) communication is being considered an important traffic offloading mechanism for future cellular networks. Coupled with pro-active device caching, it offers huge potential for capacity and coverage enhancements. In order to ensure maximum capacity enhancement, number of nodes for direct communication needs to be identified. In this paper, we derive analytic expression that relates number of D2D nodes (i.e., D2D user density) and average coverage probability of reference D2D receiver. Using stochastic geometry and poisson point process, we introduce retention probability within cooperation region and shortest distance based selection criterion to precisely quantify interference due to D2D pairs in coverage area. The simulation setup and numerical evaluation validate the closed-form expression.
\end{abstract}

Index Terms-Device-to-device communication, MPPP, retention probability, user density, average coverage probability

\section{INTRODUCTION}

$\mathbf{F}$ OR future mobile communication, huge capacity is foreseen due to growth in wireless data services. In this context, we see worldwide mobile traffic forecast of around $127 \mathrm{~EB}$ [1] and around 50 billion communication devices in 2020 [2]. In order to meet such high capacity and coverage demands, deployment of small cells (SCs) has been agile and cost-effective strategy, however, it results in global energy efficiency concerns and operational expenditure (OPEX) issues due to large number of deployments. One solution to overcome this problem is traffic off-loading to other unlicensed wireless infrastructures (e.g WiFi) or other multi-RAT networks. To this end, the MOTO project [3] proposes traffic offloading where device-to-device (D2D) communication is also considered.

D2D communication is recognized as a technology component of future 5G architecture [4]-[5]. It is considered as a horizontal topic in initial METIS D2D concept where it can provide low-power, high data rate, and low latency services in future $5 \mathrm{G}$ networks [6].

D2D communication is analogous to ad-hoc networks, however, it is based on infrastructure. In this model, the devices reuse network resources directly under little network control and virtually no involvement of core network. In this architecture, many gains are offered such as:

- Reduced path-loss may offer favorable channel conditions for most of the time, hence it may result in better signal strength and spectral efficiency.

H. A. Mustafa, M. A. Imran, and R. Tafazolli are with Center for Communication Systems Research (CCSR), University of Surrey, Guildford, UK. Emails: \{h.mustafa, m.imran, r.tafazolli\}@surrey.ac.uk

M. Z. Shakir is with Electrical and Computer Engineering Dept., Texas A\&M University at Qatar, Doha. Emails: muhammad.shakir@qatar.tamu.edu This publication was made possible by NPRP grant\# 4-1293-2-513 from Qatar National Research Fund (a member of Qatar Foundation). The statements made herein are solely the responsibility of the author[s].
- UE may act as relay network to extend coverage.

- Proximal communication offers reduced transmit power and direct communication offers no backhaul power consumption.

- Link reliability can be ensured due to single-hop topology instead of multi-hop star-like topology.

- Due to the short distance as compared to serving base station (BS) and due to single-hop topology, latency may virtually be zero.

- Core network is hardly used due to possibility of network and device pro-active caching, hence it may result in backhaul relaxation.

\section{A. Related Work}

In order to model the spatial distribution of nodes, mature framework of stochastic geometry is widely used in literature. In this framework, different nodes, (e.g., macrocells, small cells, and cellular users) are considered as point processes that produce shot-noise (SN) effect on reference point. The homogeneous poisson point process (PPP) has been extensively used in the literature [7], [8], [9] to analyze probability of successful transmission as well as transmission capacity of the system. However, in these papers, PPP has been considered to produce holistic SN effect which is valid for BS spatial distribution but not for the D2D pairs as we usually do not have all nodes to be in direct modes. Within the coverage area, only those nodes make D2D pairs which comply certain selection criterion (e.g., shortest distance). All those nodes which do not meet this criterion, they switch to the cellular mode and hence they do not cause interference at reference point.

For the case where selection criterion is not considered, it is always assumed that every node is in direct communication even if the partner node is at farthest node ${ }^{1}$. Similar to the previous papers, the authors in [10] analyzed the transmission capacity of D2D communication in heterogeneous networks assuming holistic SN effect at reference point.

\section{B. Contribution and Organization}

In this paper, we contribute the following:

- An analytic framework has been presented where average coverage probability of reference D2D pair and corresponding D2D user density in coverage area are derived. In this context, the retention probability is introduced in Laplace functional of MPPP to precisely quantify interference due to neighboring D2D nodes.

\footnotetext{
${ }^{1}$ The maximum distance between D2D pair can be twice the radius of the cell if two nodes are at extreme ends. Allowing such nodes for direct communication is not a realistic assumption.
} 
- By relaxing shortest distance criterion for D2D pairs, we derive lower bound on average coverage probability of reference D2D receiver.

The rest of the paper is organized as follows. In section II, we present system model followed by retention probability within cooperation region for D2D pairing in section III. In section IV, we analyze D2D user density and derive analytic expression for D2D user density and average coverage probability of reference D2D pair. Numerical results are presented in section $\mathrm{V}$ followed by conclusion in section VI.

\section{System ModeL}

We consider small cell base station (SBS), cellular users, reference D2D pair, and D2D interferers as shown in Fig. 1.

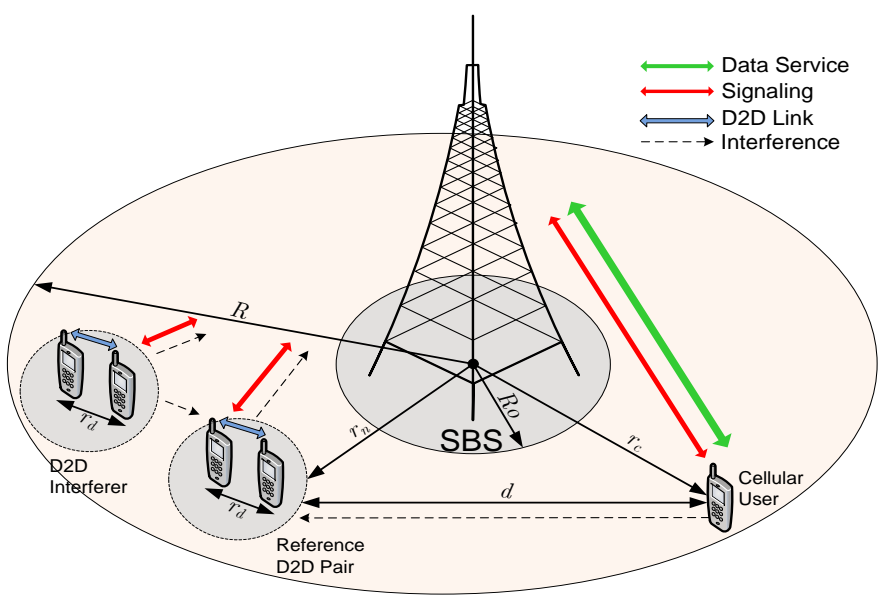

Fig. 1: System model for D2D user density.

In this system model, we assume that the potential D2D users share the uplink resources of cellular users. We consider time division duplex (TDD) mode for each D2D pair. The cellular users are provided both control and data services by the serving SBS whereas for D2D pairs, only control signaling is assumed. The interference is considered at reference D2D pair where not only neighboring D2D nodes interfere but cellular user also causes interference.

The coverage area of SBS is bounded between radius $R$ and exclusion region $R_{0}$. The distances between cellular user and SBS is $r_{c}$, whereas the distance between SBS and reference D2D pair is $r_{n}$. Every successful D2D pair has a distance of $r_{d}$ between nodes. The interference due to the cellular user on reference D2D pair is captured by distance $d$.

The SBS controls the power transmitted by the cellular user. However SBS has no power control on D2D pairs and for the sake of simplicity, it is assumed that the transmit power of D2D pairs is same. We assume distance dependent path-loss and Rayleigh fading for channels gains. The singular path-loss model $\left(r^{\alpha}\right)$ is assumed and due to exclusion region it ensures that the model converges. All received powers (whether at SBS or at reference D2D receiver) follows exponential distribution with mean 1 . The distance $r_{c}$ and $r_{n}$ follows uniform proba- bility distribution function (pdf) as follows:

$$
f(r)=\frac{2 r}{R^{2}}, f(\theta)=\frac{1}{2 \pi},
$$

where $R_{0} \leq r \leq R$ and $0 \leq \theta \leq 2 \pi$.

The distance between cellular user and D2D pair is $d$, the pdf of which is given as [11]:

$$
f(d)=\frac{2 d}{R^{2}}\left[\frac{2}{\pi} \cos ^{-1}\left(\frac{d}{2 R}\right)-\frac{d}{\pi R} \sqrt{1-\frac{d^{2}}{4 R^{2}}}\right],
$$

where $0<d \leq 2 R$.

\section{Retention Probability}

In order to quantify SN effect due to successful D2D pairs, some sort of selection criterion needs to be incorporated into Laplace functional of MPPP. If we do not consider selection criterion it means every node around the reference D2D pair is in direct mode of communication hence causing SN effect which is not a realistic assumption. To address this problem, we introduce retention probability based on shortest distance criterion to select certain nodes for D2D communication.

The homogeneous MPPP is represented by $\Phi=\left\{x_{i}, y_{i}\right\}$ where $x_{i} \in \mathbb{R}^{2}$ represent the spatial positions on the plane and $y_{i} \in \mathbb{R}^{+}$represent the associated channel gains of D2D users. In order to incorporate distance based criterion for node selection, $\Phi$ needs to be converted to $\Phi^{p}$ where $p: \mathbb{R}^{2} \mapsto[0,1]$ performs thinning of $\Phi$ to model the effect of criterion based selection of nodes.

The Laplace functional of MPPP implicitly represents the SN effect produced by number of points as [12]:

$$
\mathcal{L}_{\Phi}(f)=e^{-\int_{\mathbb{R}^{d}}\left(1-e^{-f(x)}\right) \lambda d x},
$$

where $f(x)$ is the real function defined on $\mathbb{R}^{d}$ and $\lambda$ is the intensity measure of points (i.e., D2D user density). If we introduce $p(x)$ as the retention probability to thin the number of interferers, (3) can be represented as:

$$
\mathcal{L}_{\Phi^{p}}(f)=e^{-\int_{\mathbb{R}^{d}}\left(1-e^{-f(x)}\right) p(x) \lambda d x},
$$

The probability of two points using some distance constraint is given as [13]

$$
\mathbb{P}(D<\mu)=1-e^{-\pi \lambda \mu^{2}},
$$

where $\mathrm{D}$ is the distance between two nodes and $\mu$ is the target distance.

In this paper, we modify retention probability (5) by introducing model fitting parameter $k$ as follows:

$$
p\left(r_{d}\right)=1-e^{-k \pi \lambda \mu^{2}},
$$

The model fitting parameter has been determined by simulating the system model for which the value of $k=[0.6,0.7]$ is determined as shown in Fig. 2.

In this figure, we performed model fitting for different values of $k$. It can be seen that the value of $k=[0.6,0.7]$ matches with the retention probability $p\left(r_{d}\right)$ for for different values of $\mu$. However, there are certain mismatches for higher values of $\mu$ (e.g., $\mu>100$ ). Since D2D communication should 


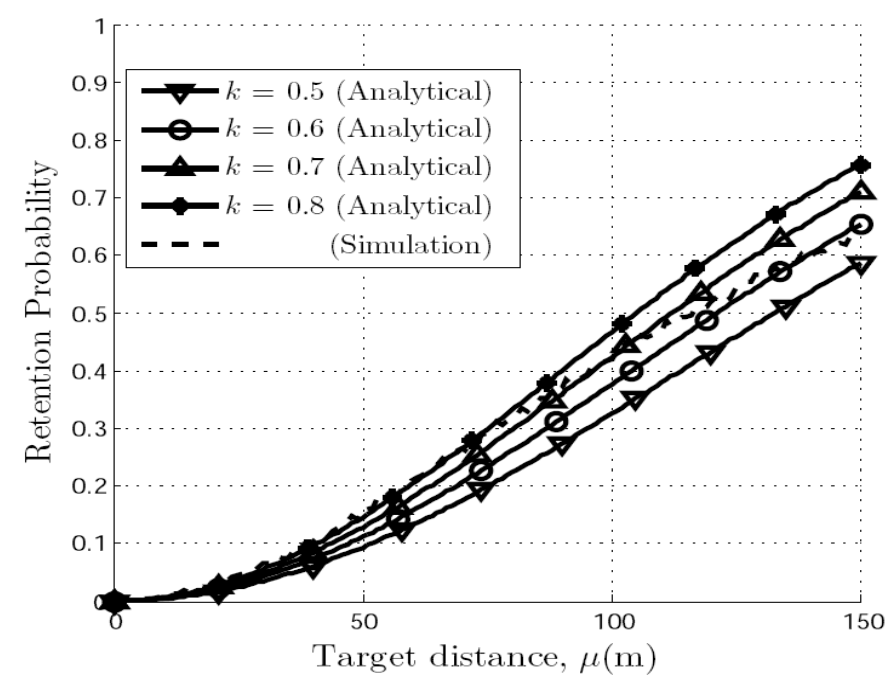

Fig. 2: Retention probability and model fitting parameter $k$ for $\lambda=0.000025$.

be considered for proximity services, it is realistic to choose small values of $\mu$ otherwise for higher values of $\mu$, normal cellular communication is more feasible. To give insight into the thinning process, we plot retention probability for a value of $k=0.7$ and different values of $\lambda$ as shown in Fig. 3 .

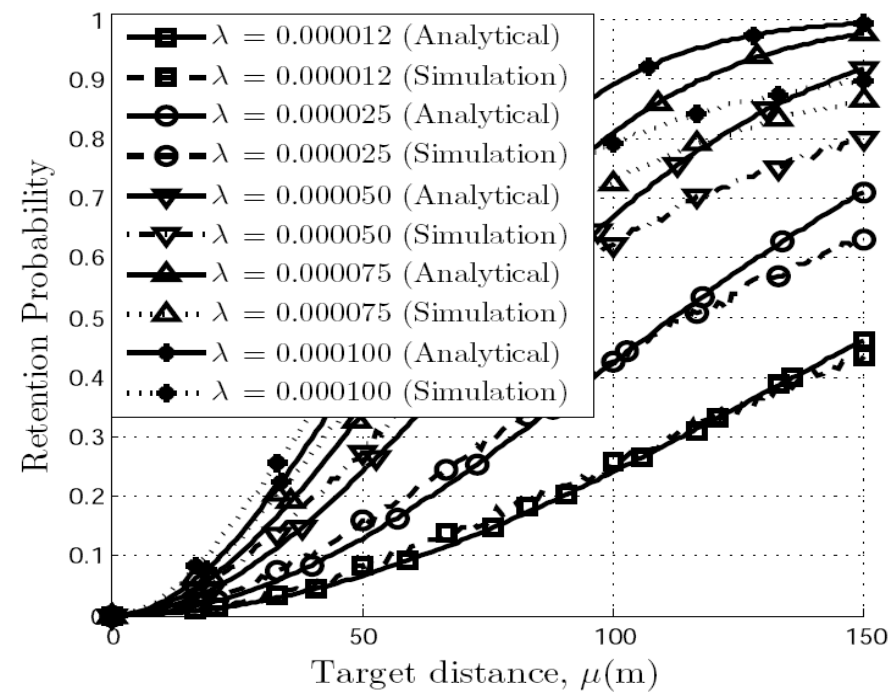

Fig. 3: Retention probability for $k=0.7$, and different $\lambda$.

In this figure, two parameters i.e., $\lambda$ and $\mu$ are important. As per intuition, higher value of $\lambda$ means more D2D interferers and large values of $\mu$ means higher path-loss between D2D pairs. From these curves, we can see that there is a tradeoff between $\lambda$ and $\mu$. The lower value of $\lambda$ (e.g., 0.000012) offers higher $\mu$ (e.g., 150m) whereas higher values of $\lambda$ (e.g., 0.0001 ) reduces $\mu$ to $85 \mathrm{~m}$ for a close match between analytic expression and simulation setup. In this paper, we have chosen $k=0.7$ with $\mu=100 \mathrm{~m}$ as maximum distance for D2D pairing.

\section{D2D USER DENSITY}

In this section, we derive average coverage probability of reference D2D pair in terms of D2D user density $\lambda$. We consider interference-limited environment $\left(\sigma^{2}=0\right)$, hence signal-to-interference ratio (SIR) for reference pair is given as follows:

$$
\operatorname{SIR}_{k}=\frac{p_{d} f_{d} r_{d}^{-\alpha}}{p_{c} f_{c} d^{-\alpha}+\sum_{i \in \Phi \backslash k} p_{i} f_{i} r_{i}^{-\alpha}},
$$

For brevity in analytic derivations in Appendix A, the numerators and denominators of (7) are represented as

$$
\begin{gathered}
I_{d}=p_{d} f_{d} r_{d}^{-\alpha}, \\
I_{c}=p_{c} f_{c} d^{-\alpha}, \\
I_{n}=\sum_{i \in \Phi \backslash k} p_{i} f_{i} r_{i}^{-\alpha},
\end{gathered}
$$

where $I_{d}$ is the received power between reference D2D transmitter and receiver, $I_{c}$ is the interference due to cellular user on reference D2D receiver, and $I_{n}$ is the accumulated received power of all interferring D2D pairs. In (8), the received power comprises of transmit power $p_{d}$ of reference D2D transmitter, small-scale fading $f_{d}$, and distance dependent path-loss $r_{d}^{-\alpha}$. In (9) and (10), the received power comprises of transmit power $p_{c}$ of interfering cellular user and transmit power $p_{i}$ of all interfering D2D pairs, respectively. The corresponding small-scale fading and distance dependent pathlosses are given as $f_{c}, f_{i}, d^{-\alpha}$, and $r_{i}^{-\alpha}$, respectively.

The total interference experienced by a single D2D receiver $k$ is the sum of interference from primary cellular user and all other D2D transmitters. Using (7) and assuming exponential distribution for desired and interfering channels $f_{x}$ for $x \in$ $\{d, c, i\}$, the average coverage probability of D2D receiver $k$ is given by the following theorem.

Theorem 1. The average coverage probability of an underlay $D 2 D$ receiver is

$$
p_{\text {cov }}^{d} \simeq \int_{R_{0}}^{R} \frac{e^{-\frac{2 \pi^{2} \lambda\left(1-e^{\left.-k \pi \lambda \mu^{2}\right) r_{d}^{2}}\right.}{\alpha \sin \left(\frac{2 \pi}{\alpha}\right)}\left(\frac{\gamma}{p_{d}}\right)^{\frac{2}{\alpha}} \mathbb{E}\left[p_{i}^{\frac{2}{\alpha}}\right]} 2 r_{n}}{1+\left(\gamma \frac{p_{c}}{p_{d}}\right)^{\frac{2}{\alpha}} \frac{r_{d}^{2}}{\left(\frac{128 \pi}{45 \pi}\right)^{2}}} d r_{n},
$$

Proof: See Appendix A.

The transmit power of reference D2D pair and all other interfering D2D nodes is assumed to be same $\left(p_{i}=p_{d}\right)$. For $R_{0} \sim 0$, and $\alpha=4$, the average coverage probability (11) reduces to:-

$$
p_{\text {cov }}^{d} \simeq \frac{e^{-\frac{\pi^{2} r_{d}^{2} \lambda}{2} \sqrt{\gamma}\left(1-e^{-k \pi \lambda \mu^{2}}\right)}}{1+\sqrt{\gamma \frac{p_{c}}{p_{d}}} \frac{r_{d}^{2}}{\left(\frac{128 R}{45 \pi}\right)^{2}}},
$$

The lower bound on average coverage probability of reference D2D receiver can be derived by assuming same transmit power for every D2D pair, and retention probability $p\left(r_{d}\right)=1$ (i.e., allowing every node to be in D2D pair).

Corollary 1.1. The lower bound on average coverage probability of reference D2D pair for same power $p_{i}$ and $p\left(r_{d}\right)=1$ 
is given as follows:

$$
p_{c o v, l b}^{d} \simeq \frac{e^{-\frac{\pi^{2} r_{d}^{2} \lambda}{2}} \sqrt{\gamma}}{1+\sqrt{\gamma \frac{p_{c}}{p_{d}}} \frac{r_{d}^{2}}{\left(\frac{128 R}{45 \pi}\right)^{2}}},
$$

\section{Numerical Results}

In this section, we numerically evaluate the analytic expressions of Sec. IV by varying the number of different parameters for a fixed cell of radius $R=500 \mathrm{~m}$ and a radius of protection region $R_{0}=1 \mathrm{~m}$. The cell edge effect is simulated by dropping D2D nodes around cell boundary. The D2D pairs are chosen on shortest distance criterion which is captured analytically by thinning the poisson point process using retention probability (6). The power ratio of cellular user and D2D transmitter is assumed to be 500 with $p_{c}=100 \mathrm{~mW}$ and $p_{i}=0.2 \mathrm{~mW}$. In order to calculate the average effect of coverage probability, 3000 monte-carlo simulations are run. For an average coverage probability of a reference D2D receiver, a single drop of D2D pairs for $\lambda=0.000075$ is shown in Fig. 4 .

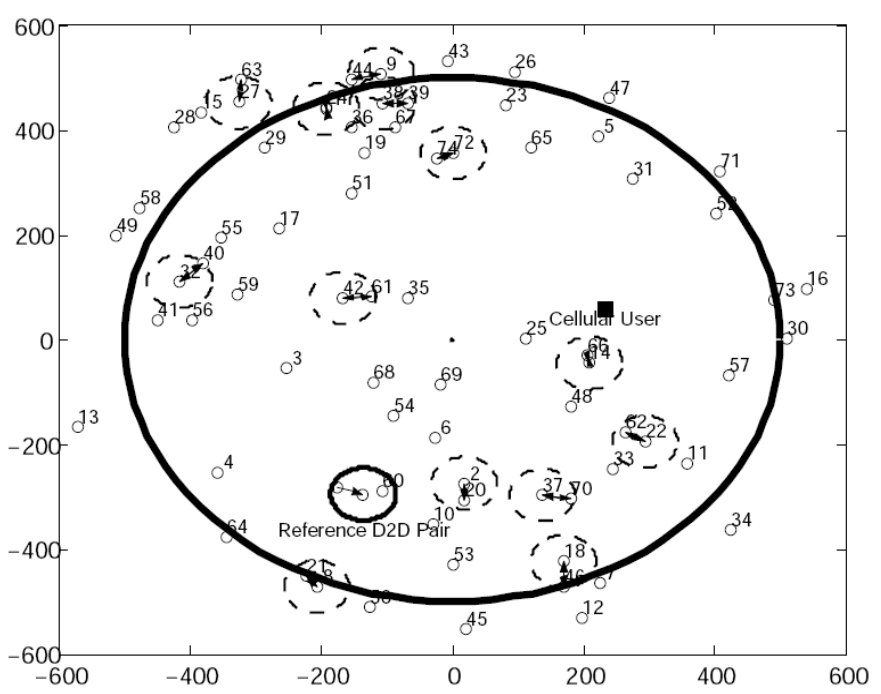

Fig. 4: Single drop of D2D pairs for evlauation of theorem $1(\lambda=0.000075$, $r_{d}<50 \mathrm{~m}$, and $\left.\mu=50 \mathrm{~m}\right)$.

In this figure, solid square represents a cellular user, dotted small circles represent successful D2D pairs that produce SN effect and solid small circle represents reference D2D pair where the average coverage probability of D2D receiver is evaluated. For the setup of Fig. 4, the average coverage probability of a reference $\mathrm{D} 2 \mathrm{D}$ receiver is calculated as shown in Fig. 5.

The distance between reference D2D transmitter and receiver has been assumed to be $30 \mathrm{~m}$. The coverage trends for different values of $\lambda$ are very similar. Although the coverage probability of D2D receiver has been evaluated for a range of $\gamma$, however it is intuitive and instructive to use very small radius $\mu$ to perform D2D pairing (shortest distance requires less transmit power and long battery time). Therefore, lower values of $\gamma$ are required between D2D nodes and hence higher coverage probability is ensured. For example, the coverage

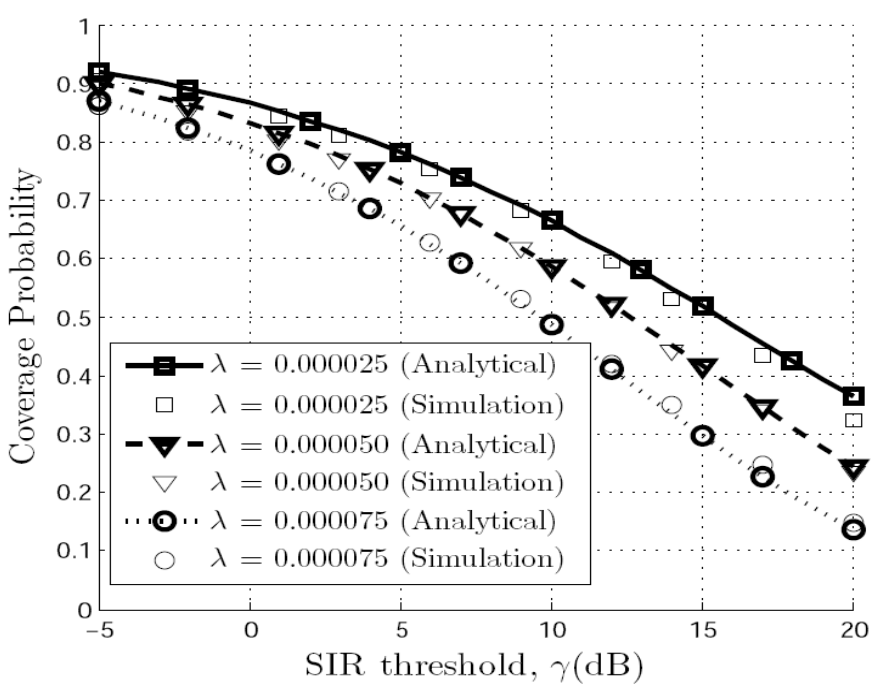

Fig. 5: Average coverage probability of reference D2D pair for $p_{i}=0.2 \mathrm{~mW}$, $p_{c}=100 \mathrm{~mW}, r_{d}<50 \mathrm{~m}$, and $\mu=50 \mathrm{~m}$.

drop for $\lambda=[0.000025,0.000075]$ is around $8 \%$ which is not very significant as compared to increase (3 times in this case) in D2D user density. The curves at lower values of $\gamma$ show better coverage because of the dependence of coverage probability on distance between reference transmitter and receiver. In case of reference D2D pair, this distance is much smaller as compared to the distance between cellular user and serving SBS.

The lower bound on average coverage probability of reference D2D pair is shown in Fig. 6.

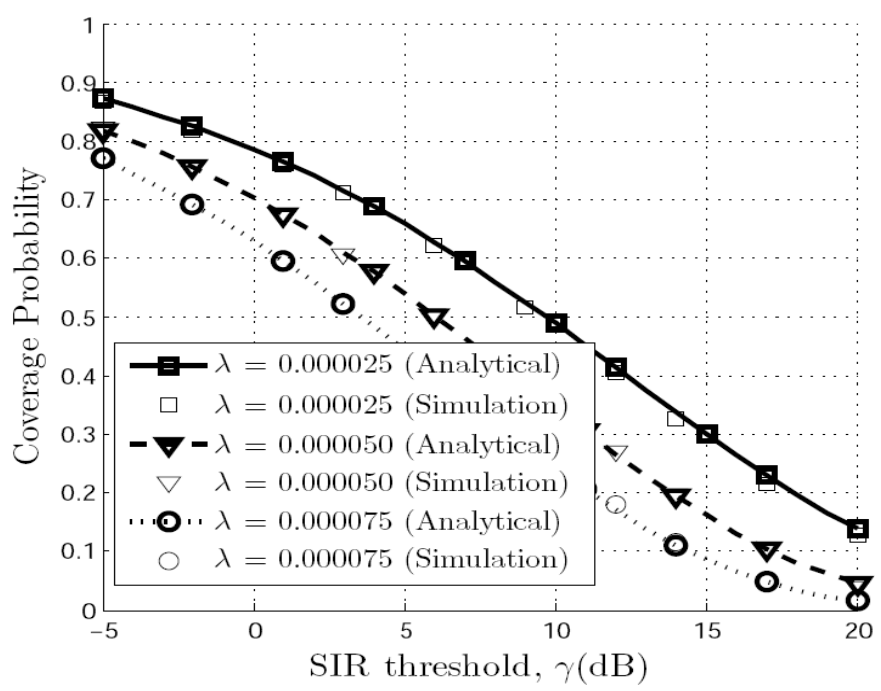

Fig. 6: Lower bound on average coverage probability of reference D2D pair for $R=500 \mathrm{~m}, p_{i}=0.2 \mathrm{~mW}, p_{c}=100 \mathrm{~mW}, r_{d}<50 \mathrm{~m}$, and $\mu=50 \mathrm{~m}$.

Although a non-realistic scenario has been assumed where every D2D node is in direct communication with the partner node, however, for an equal D2D transmit power, the lower bound can be evaluated to analyze the measure of interference generated by every node. The coverage trend is rather smooth and meets the intuition of coverage drop due to increased 
number of D2D pairs.

The effect of thinning and no-thinning of MPPP has been shown in Fig. 7. In case of no-thinning, the analytic expression

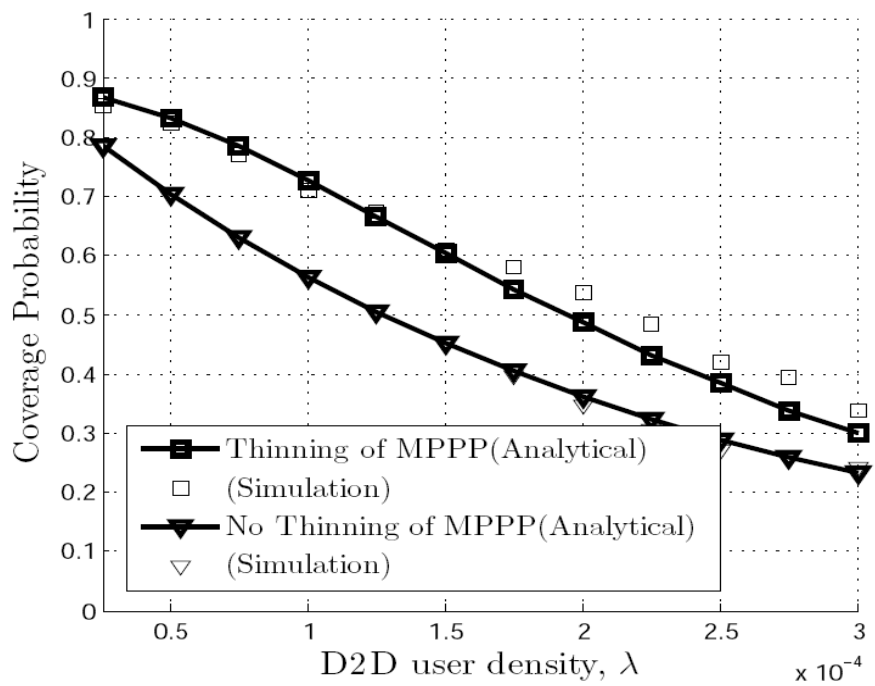

Fig. 7: Average coverage probability of reference D2D pair for variable $\lambda$, $r_{d}<50 \mathrm{~m}$, and $\mu=50 \mathrm{~m}$.

perfectly matches with the simulation setup whereas thinning process produces certain mismatch for higher values of $\lambda$. This effect is in compliance with curves of retention probability (Fig. 3).

\section{Vi. CONCLUSIONS}

In this paper, we introduced retention probability (i.e., shortest distance based selection process) in Laplace functional of MPPP to identify D2D pairs in the coverage area. This process performed thinning of point process and precisely model the SN effect due to D2D pairs on reference D2D receiver. Based on this spatial distribution of D2D nodes, the average coverage probability of reference D2D pair has been analytically and numerically evaluated. The simulation results verify the closed-form approximations for different values of $\lambda$, and $\gamma$. By relaxing selection criterion i.e., $p\left(r_{d}\right)=1$, a lower bound on average coverage probability has also been derived.

It has been observed that there is a certain mismatch between analytic expression of retention probability and the simulation step which is due to the non-exclusion of nodes within the radius of potential D2D pairs. This non-exclusion phenomenon has not been captured completely in analytic expression of retention probability. However, assuming point to multi-point $\mathrm{D} 2 \mathrm{D}$ pairs within cooperation radius, this mismatch can be reduced.

\section{ACKNOWLEDGEMENTS}

We would like to acknowledge the support of the University of Surrey 5GIC (http://www.surrey.ac.uk/5gic) members for this work. Moreover, we acknowledge the support of Dr. Shakir under NPRP grant \# 4-1293-2-513 from Qatar National Research Fund.

\section{APPENDIX A}

PROOF OF THEOREM 1

The average coverage probability of $\mathrm{D} 2 \mathrm{D}$ receiver $k$, distributed uniformly over plane between $R$ and $R_{0}$ (at a distance $d$ from the cellular interferer), conditioned on $g=\left[p_{i}, f_{i}\right]$ and $h=\left[p_{c}, d, f_{c}\right]$ is given as follows:

$$
\begin{aligned}
p_{\text {cov }}^{d} & =\mathbb{E}_{r_{n}, h, g}\left[\mathbb{P}\left[\operatorname{SIR}_{k} \geq \gamma\right] \mid r_{n}, h, g\right], \\
& =\mathbb{E}_{r_{n}, h, g}\left[\mathbb{P}\left[\left(f_{d} \geq \gamma \frac{I_{d}+I_{n}}{p_{d} r_{d}^{-\alpha}}\right)\right] \mid r_{n}, h, g\right],
\end{aligned}
$$

The conditional coverage probability from (14) is evaluated as:

$$
\begin{aligned}
\mathbb{P}\left[\left(f_{d} \geq \gamma \frac{I_{d}+I_{n}}{\left.\left.p_{d} r_{d}^{-\alpha}\right)\right] \mid r_{n}, h, g}\right.\right. & =\int_{x=\gamma \frac{I_{d}+I_{n}}{p_{d} r_{d}^{-\alpha}}}^{\infty} e^{-x} d x, \\
& =e^{-\frac{\gamma\left(I_{d}+I_{n}\right)}{p_{d} r_{d}^{-\alpha}}} \\
& =e^{-\frac{\gamma I_{d}}{p_{d} r_{d}^{-\alpha}}} e^{-\frac{\gamma I_{n}}{p_{d} r_{d}^{-\alpha}}},
\end{aligned}
$$

Putting (15) in (14),

$$
\begin{aligned}
p_{\text {cov }}^{d} & =\mathbb{E}_{r_{n}, h, g}\left[e^{-\frac{\gamma I_{d}}{p_{d} r_{d}^{-\alpha}}} e^{-\frac{\gamma I_{n}}{p_{d} r_{d}^{-\alpha}}}\right], \\
& =\mathbb{E}_{r_{n}}\left[\mathbb{E}_{h}\left[e^{-\frac{\gamma I_{d}}{p_{d_{d}}-\alpha}}\right] \mathbb{E}_{g}\left[e^{-\frac{\gamma I_{n}}{p_{d} r_{d}^{-\alpha}}}\right]\right], \\
& =\mathbb{E}_{r_{n}}\left[\mathcal{L}_{I_{d}}\left(s_{d}\right) \mathcal{L}_{I_{n}}\left(s_{d}\right)\right],
\end{aligned}
$$

where $s_{d}=\gamma p_{d}^{-1} r_{d}^{\alpha}$. De-conditioning (by $h=\left[p_{c}, d, f_{c}\right]$ ) of the first part on right hand side of (16) and putting the value of $I_{d}$ from (8) results:

$$
\mathcal{L}_{I_{d}}\left(s_{d}\right)=\mathbb{E}_{p_{c}, d, f_{c}}\left[e^{-s_{d} p_{c} f_{c} d^{-\alpha}}\right],
$$

where the expectation considers cellular user distributions for uplink power, distance, and channel gain to the reference D2D receiver. In order to get insight into (17), we assume 1) the transmit power of cellular interferer is independent of D2D interferers and is controlled only by the serving SBS), 2) distance $d$ between cellular interferer and reference D2D receiver follows pdf given by (2), and 3) all fading channels follow $\operatorname{Exp}(1)$. The resulting cellular interference is

$$
\begin{aligned}
\mathcal{L}_{I_{d}}\left(s_{d}\right) & =\mathbb{E}_{d}\left[\mathbb{E}_{f_{c}}\left[e^{-s_{d} p_{c} f_{c} d^{-\alpha}}\right]\right], \\
& =\mathbb{E}_{d}\left[\frac{1}{1+s_{d} p_{c} d^{-\alpha}}\right], \\
& =\mathbb{E}_{d}\left[\frac{1}{1+\gamma \frac{p_{c} r_{d}^{\alpha}}{p_{d} d^{\alpha}}}\right],
\end{aligned}
$$

Using approximation from [9] [Sec. IV-C] and the first moment of $d$ from [11], (18) results into

$$
\mathcal{L}_{I_{d}}\left(s_{d}\right) \simeq \frac{1}{1+\left(\gamma \frac{p_{c}}{p_{d}}\right)^{\frac{2}{\alpha}} \frac{r_{d}^{2}}{(128 R / 45 \pi)^{2}}},
$$

All interferers (D2D transmitters) are dropped according to 
MPPP; therefore, second part on right hand side of (16) is:

$$
\begin{aligned}
\mathcal{L}_{I_{n}}\left(s_{d}\right) & =\mathbb{E}_{\Phi, p_{i}, f_{i}}\left[e^{-s_{d} \sum_{i \in \Phi \backslash k} p_{i} f_{i} r_{i}^{-\alpha}}\right] \\
& =\mathbb{E}_{\Phi, p_{i}, f_{i}}\left[\prod_{i \in \Phi \backslash k} e^{-s_{d} p_{i} f_{i} r_{i}^{-\alpha}}\right] \\
& =\mathbb{E}_{\Phi, p_{i}}\left[\prod_{i \in \Phi \backslash k} \mathbb{E}_{f_{i}}\left[e^{-s_{d} p_{i} f_{i} r_{i}^{-\alpha}}\right]\right] \\
& =\mathbb{E}_{\Phi}\left[\prod_{i \in \Phi \backslash k} \mathbb{E}_{p_{i}}\left(\frac{1}{1+s_{d} p_{i} r_{i}^{-\alpha}}\right)\right] \\
& =\mathbb{E}_{\Phi}[\prod_{i \in \Phi \backslash k} \underbrace{\left(\frac{1}{1+s_{d} \mathbb{E}\left[p_{i}\right] r_{i}^{-\alpha}}\right)}_{f(x)}]
\end{aligned}
$$

where (20) results from the i.i.d distributions of $p_{i}$ and $f_{i}$ and further independence from underlay MPPP process. The probability generating functional (PGFL) for a function $f(x)$ with retention probability $p\left(r_{d}\right)$ from (6) implies:

$$
\begin{aligned}
\mathbb{E}\left[\prod_{m \in \Phi \backslash k} f(x)\right] & =e^{-\int_{\mathbb{R}^{2}}(1-f(x)) p\left(r_{d}\right) \lambda d x}, \\
& =e^{-\lambda p\left(r_{d}\right) \int_{0}^{\infty} \int_{0}^{2 \pi}(1-f(x)) x d r d x}, \\
& =e^{-2 \pi \lambda p\left(r_{d}\right) \int_{0}^{\infty}(1-f(x)) x d x},
\end{aligned}
$$

Putting $f(x)$ from (20) into (21) results:

$$
\begin{aligned}
\mathcal{L}_{I_{n}}\left(s_{d}\right) & =e^{-2 \pi \lambda p\left(r_{d}\right) \int_{R_{0}}^{\infty}\left(1-\frac{1}{1+s_{d} \mathbb{E}\left[p_{i}\right] x-\alpha}\right) x d x}, \\
& =e^{-2 \pi \lambda p\left(r_{d}\right) \int_{R_{0}}^{\infty}\left(\frac{s_{d} \mathbb{E}\left[p_{i}\right] x^{-\alpha}}{1+s_{d} \mathbb{E}\left[p_{i}\right] x^{-\alpha}}\right) x d x}, \\
& =e^{-2 \pi \lambda p\left(r_{d}\right) \int_{R_{0}}^{\infty}\left(\frac{1}{1+\frac{x^{\alpha}}{s_{d} \mathbb{E}\left[p_{i}\right]}}\right) x d x},
\end{aligned}
$$

By substituting $\frac{x^{\alpha}}{s_{d} \mathbb{E}\left[p_{i}\right]}=u^{\alpha},(22)$ results

$$
\mathcal{L}_{I_{n}}\left(s_{d}\right)=e^{-2 \pi \lambda p\left(r_{d}\right)\left(s_{d}\right)^{\frac{2}{\alpha}} \mathbb{E}\left[p_{i}^{\frac{2}{\alpha}}\right] \int_{R_{0}}^{\infty}\left(\frac{u}{1+u^{\alpha}}\right) d u},
$$

Since $R_{0} \ll R$, therefore assuming $R_{0} \sim 0$, the integral on right hand side of (23) can be evaluated as:

$$
\int_{0}^{\infty}\left(\frac{u}{1+u^{\alpha}}\right) d u=\frac{\pi}{\alpha \sin \left(\frac{2 \pi}{\alpha}\right)},
$$

Putting (24) into (23) results into:

$$
\mathcal{L}_{I_{n}}\left(s_{d}\right)=e^{-\frac{2 \pi^{2} \lambda p\left(r_{d}\right) r_{d}^{2}}{\alpha \sin \left(\frac{2 \pi}{\alpha}\right)}\left(\frac{\gamma}{p_{d}}\right)^{\frac{2}{\alpha}} \mathbb{E}\left[p_{i}^{\frac{2}{\alpha}}\right]},
$$

Putting (19) and (25) into (16), the average coverage probability of $\mathrm{D} 2 \mathrm{D}$ receiver $k$ is:

$$
\begin{aligned}
p_{\text {cov }}^{d} & \simeq \mathbb{E}_{r_{n}}\left[\mathcal{L}_{I_{d}}\left(s_{d}\right) \mathcal{L}_{I_{n}}\left(s_{d}\right) \mid r_{n}\right] \\
& \simeq \int_{R_{0}}^{R} \frac{e^{-\frac{2 \pi^{2} \lambda\left(1-e^{-k \pi \lambda \mu^{2}}\right) r_{d}^{2}}{\alpha \sin \left(\frac{2 \pi}{\alpha}\right)}\left(\frac{\gamma}{p_{d}}\right)^{\frac{2}{\alpha}} \mathbb{E}\left[p_{i}^{\frac{2}{\alpha}}\right]}}{1+\left(\gamma \frac{p_{c}}{p_{d}}\right)^{\frac{2}{\alpha}} \frac{r_{d}^{2}}{\left(\frac{128 R}{45 \pi}\right)^{2}}} \frac{2 r_{n}}{R^{2}} d r_{n},
\end{aligned}
$$

\section{REFERENCES}

[1] "UMTS Forum Report: Mobile Traffic Forecasts: 20102020," Report 44, May 2011. [Online]. Available: http://www.umts-forum.org/component/

[2] D. Evans, "The Internet of Things, How the Next Evolution of the Internet is Changing Everything," Cisco, White Paper, Apr. 2011. [Online]. Available: http://www.cisco.com/web/about/ac79/docs/ innov/IoT_IBSG_0411FINAL.pdf

[3] "Radio Access and Spectrum FP7 - Future Networks Cluster." [Online]. Available: http://www.ict-ras.eu/ index.php/ras-projects/moto

[4] "D2D Communications What Part Will It Play in 5G?" [Online]. Available: http://www.ericsson.com/ research-blog/5g/device-device-communications/

[5] "5G radio network architecture." [Online]. Available: http://fp7-semafour.eu/media/cms_page_media/9/ SEMAFOUR_2014_RAScluster

[6] Z. Li, M. Moisio, M. A. Uusitalo, P. Lundén, C. Wijting, F. S. Moya, A. Yaver, and V. Venkatasubramanian, "Overview on initial METIS D2D Concept."

[7] X. Xu, J. Sun, and S. Shao, "Transmission Capacity Of D2D Communication Under Cellular Networks." Atlantis Press, 2013. [Online]. Available: http://www. atlantis-press.com/php/paper-details.php?id=6550

[8] A. H. Sakr and E. Hossain, "Cognitive and energy harvesting-based D2D communication in cellular networks: stochastic geometry modeling and analysis," May 2014. [Online]. Available: http://arxiv.org/abs/1405.2013

[9] N. Lee, X. Lin, J. G. Andrews, and R. W. Heath Jr, "Power Control for D2D Underlaid Cellular Networks: Modeling, Algorithms and Analysis," May 2013. [Online]. Available: http://arxiv.org/abs/1305.6161

[10] Z. Liu, H. Chen, T. Peng, and W. Wang, "Optimal density and power allocation of D2D communication under heterogeneous networks on multi-bands with outage constraints," in Computing, Networking and Communications (ICNC), 2013 International Conference on, Jan 2013, pp. 1179-1183.

[11] D. Moltchanov, "Distance distributions in random networks," Ad Hoc Networks, vol. 10, no. 6, pp. 1146-1166, 2012.

[12] B. Blaszczyszyn, Stochastic Geometry and Wireless Networks, Part I: Theory. Now Publishers Inc.

[13] X. Lin, J. G. Andrews, and A. Ghosh, "Spectrum Sharing for Device-to-Device Communication in Cellular Networks," May 2013. [Online]. Available: http://arxiv. org/abs/1305.4219 